\title{
Dark on a light subject
}

\section{Nineteenth-century physicists couldn't agree about the speed of light.}

\section{Brian Pippard}

The six volumes of the English mathematician and physicist Lord Rayleigh's collected papers record a life's work unusually free from mistakes. One paper of 1881 , however, is partly omitted as erroneous. This paper was the opening shot in a good-humoured conflict about the speed of light among leading physicists in Britain and America, with a little help from France - most is told as letters to Nature.

We must first go back 30 years, to 1851 and the determinations of the speed of light by Armand Fizeau and Jean Foucault in Paris. Fizeau used a rapidly spinning toothed wheel to chop light into short sections which were reflected from a mirror $8.6 \mathrm{~km}$ away; they took long enough to return so that, with the wheel spinning at certain speeds, they were obscured by a tooth. Foucault reflected light from a spinning mirror and thence to a distant fixed mirror, so that what returned found the spinning mirror had turned to displace the image.

What concerns us here is the repetition of Fizeau's measurement by James Young and George Forbes of Glasgow, who reported in 1881 that blue light travels $1.8 \%$ faster than red, and Rayleigh's comment on their work. Their result was more credible in Glasgow than elsewhere, for Lord Kelvin did not quite accept James Clerk Maxwell's theory of light as electromagnetic waves and was (rightly) satisfied that Young and Forbes were capable experimentalists. Elsewhere, however, there were sceptics, particularly among those astronomers who had never observed a celestial body displaying a succession of colours as it emerged from eclipse. Across the Atlantic, Albert Abraham Michelson, an old hand at the speed-of-light business, wrote that in his measurements the effect would have spread the image of the source slit into a spectrum.

Michelson had written within days of reading Rayleigh's critique of Young and Forbes, which raised the question of what speed is actually measured. In his Theory of Sound (1877) Rayleigh had shown that a limited train of waves, such as that produced by Fizeau's wheel, would travel in a dispersive medium at a speed, $u$, different from that of the individual wave crests, $v$. If the wavenumber ( $2 \pi /$ wavelength) is $k$ and the angular frequency is $\omega$, then $v=\omega / k$ and $u=\mathrm{d} \omega / \mathrm{d} k$. The results of Young and Forbes would imply that $v$ is $2.7 \%$ less than $u$, and this is inconsistent with the value of $v$ that alone comes from measurement of the aber- ration of stars. All other determinations, said Rayleigh, are of $u$.

This remark started a new hare when Rayleigh was asked to explain his belief that Foucault's method measured the speed of light as $u$. He obliged, but had to admit that he had overlooked a complication. When light is reflected from a spinning mirror, one side is moving towards the light source, and the other side away. There is therefore a different Doppler shift from the two sides, so that the outgoing wavelength varies across the width of the mirror, and in a dispersive medium the two sides of the wavefront move at different speeds, and the wavefront tilts during its long journey. This results in an extra displacement of the image, so that it is neither $v$ nor $u$ that is measured, but $v^{2} / u$. He added that a convex lens in front of the fixed mirror would invert the wavefront and allow the extra tilt to be cancelled, so that now $u$ would be measured after all.

Nothing much happened for five years, until Arthur Schuster wrote from Manchester to dispute George Gouy’s opinion, “given

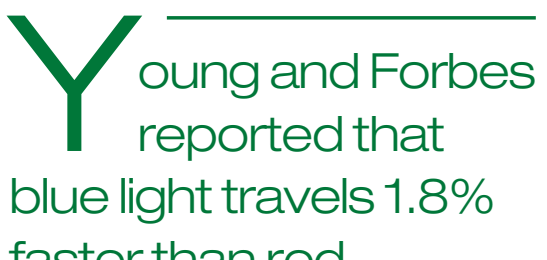

with insufficient reason", that $u$ is correct, and to question Rayleigh's analysis of the effect of a convex lens; it wouldn't cancel the Doppler effect, but enhance it, to give the measured speed as $v^{2} /(2 v-u)$. Schuster says that Rayleigh concurs, but now Willard Gibbs of Yale enters the ring, moved by Schuster's letter to dispose of the whole matter in magisterial fashion: "I am not aware that attention has been called to the important fact, that while the individual wave rotates the wavefront of the group remains unchanged... To get a picture of the phenomenon, we may imagine that we are able to see a few inches of the top of a moving carriage wheel. The individual spokes rotate, while the group maintains a vertical direction." The right answer, after all, is $u$, and Gibbs fires a Parthian shot at Schuster - if he hadn't made a false assumption he too would have got $u$.

This closes the discussion, with honours to America - Michelson and Gibbs spot on; and the British team of Young and Forbes, Kelvin, Rayleigh and Schuster all a little offbeam. But what went wrong with the Glasgow measurements? It is hard to fault the very full account they gave in 1882 of their prolonged and scrupulous research. All the same, it was vitiated by an unexplained effect and, like many another honest error, has been consigned to oblivion.

Brian Pippard is at the Cavendish Laboratory, Department of Physics, University of Cambridge, Madingley Road, Cambridge CB3 OHE, UK.

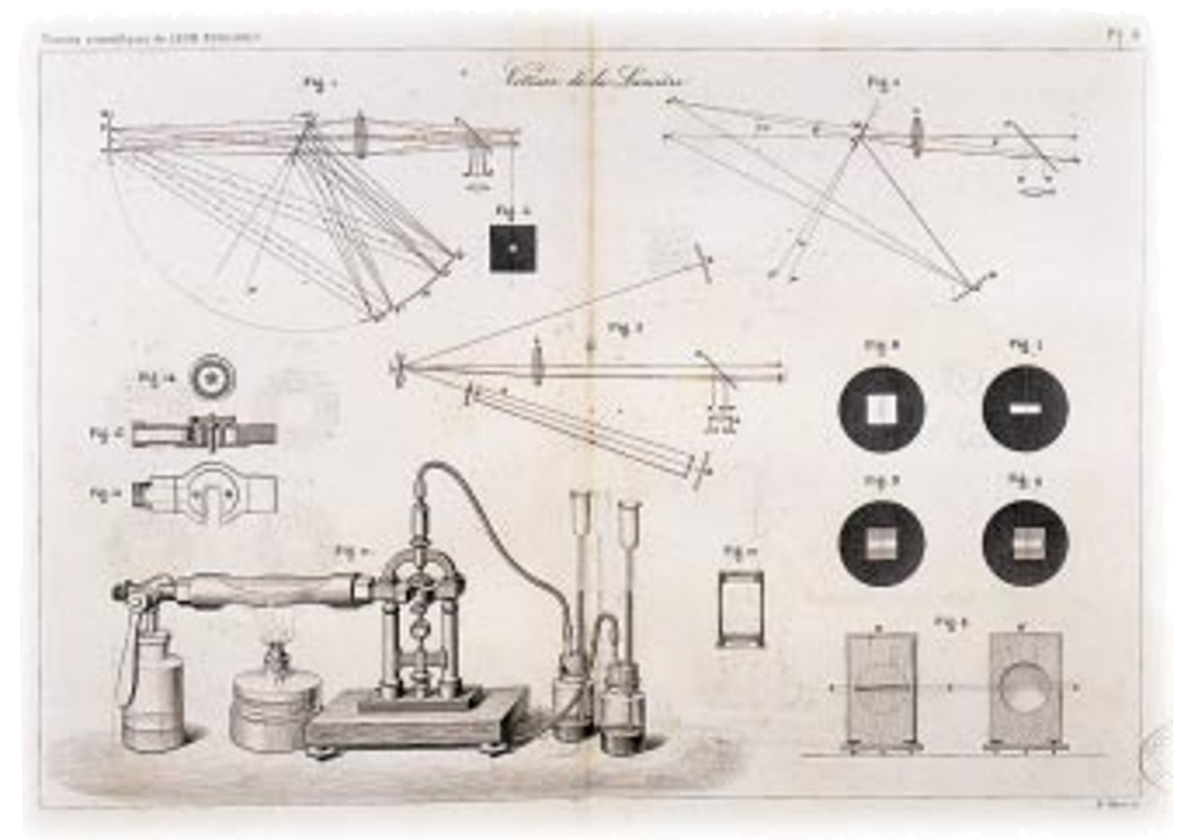

It's all done with mirrors: Foucault's apparatus for measuring the speed of light. 